\title{
Baryon number fluctuations in quasi-particle model
}

\author{
Ameng Zhao ${ }^{1, \mathrm{a}}$, Xiaofeng Luo ${ }^{2, \mathrm{~b}}$, Hongshi Zong ${ }^{3,4,5, \mathrm{c}}$ \\ ${ }^{1}$ Department of Foundation, Southeast University Chengxian College, Nanjing 210088, China \\ ${ }^{2}$ Key Laboratory of Quark and Lepton Physics (MOE), Institute of Particle Physics, Central China Normal University, Wuhan 430079, China \\ ${ }^{3}$ Department of Physics, Nanjing University, Nanjing 210093, China \\ ${ }^{4}$ Joint Center for Particle, Nuclear Physics and Cosmology, Nanjing 210093, China \\ ${ }^{5}$ State Key Laboratory of Theoretical Physics, Institute of Theoretical Physics, CAS, Beijing 100190, China
}

Received: 7 September 2016 / Accepted: 23 March 2017 / Published online: 1 April 2017

(C) The Author(s) 2017. This article is an open access publication

\begin{abstract}
Baryon number fluctuations are sensitive to the QCD phase transition and the QCD critical point. According to the Feynman rules of finite-temperature field theory, we calculated various order moments and cumulants of the baryon number distributions in the quasi-particle model of the quark-gluon plasma. Furthermore, we compared our results with the experimental data measured by the STAR experiment at RHIC. It is found that the experimental data can be well described by the model for the colliding energies above $30 \mathrm{GeV}$ and show large discrepancies at low energies. This puts a new constraint on the qQGP model and also provides a baseline for the QCD critical point search in heavy-ion collisions at low energies.
\end{abstract}

\section{Moments of net-baryon distributions and quasi-particle model of QGP}

Lattice QCD calculations indicate that at baryon chemical potential $\mu_{\mathrm{B}}=0$, the transition from the quark-gluon plasma (QGP) to a hadron gas is a smooth crossover, while at large $\mu_{\mathrm{B}}$, the phase transition is of first order. The end point of the first order phase transition boundary is the socalled the critical point (CP). The fluctuations of the netproton number measured by the STAR experiment at RHIC suggest that a possible $\mathrm{CP}$ is unlikely below $\mu_{\mathrm{B}}=200$ $\mathrm{MeV}$ [1]. The moments of the conserved quantities distributions, for example the net-baryon number, in the relativistic heavy ion collisions are sensitive to the correlation length $\xi$ of the system [2,3], and they are believed to be good signatures of the QCD phase transition and CP. Typically variances $\left(\sigma^{2}=\left\langle(N-\langle N\rangle)^{2}\right\rangle\right)$ of the distributions

\footnotetext{
a e-mail: zhaoameng@cxxy.seu.edu.cn

b e-mail: xfluo@mail.ccnu.edu.cn

c e-mail: zonghs@nju.edu.cn
}

are related to $\xi$ by $\sigma^{2} \sim \xi^{2}$. The numerator in the skewness $\left(S=\left\langle(N-\langle N\rangle)^{3}\right\rangle / \sigma^{3}\right)$ goes as $\xi^{4.5}$ and the kurtosis $\left(\kappa=\left\langle(N-\langle N\rangle)^{4}\right\rangle / \sigma^{4}-3\right)$ goes as $\xi^{7}$.

On the other hand, the moments of the baryon number are related to the various order baryon number susceptibilities [4]. In order to cancel the volume, the products of the moments, $S \sigma$ and $\kappa \sigma^{2}$, are constructed as the experimental observables. The results in RHIC of these observables show a centrality and energy dependence [5], which are not reproduced by a non-CP transport and hadron resonance gas model calculations. The deviations of $S \sigma$ and $\kappa \sigma^{2}$ below the Skellam expectation are qualitatively consistent with a QCDbased model which includes a CP [6]. The energy dependence of the $\kappa \sigma^{2}$ of net-proton distributions in $\mathrm{Au}+\mathrm{Au}$ collisions show non-monotonic behavior, which is consistent with being close to the CP $[7,8]$.

In this paper we apply the quasi-particle model (qQGP) of the quark-gluon plasma (QGP) to calculate the moments of the net-baryon distributions. The qQGP model was first proposed by Peshier et al. [9] to study the non-ideal equation of state (EoS) by lattice QCD results. Instead of real quarks and gluons with QCD interactions, the system is considered to be made up of non-interacting quasi-quarks and quasigluons with thermal masses. Quasi-particles are thought to be quanta of plasma collective modes excited by quarks and gluons through QCD interactions.

By now, some approaches have been proposed to study the qQGP model. The effective mass methods [9-14], the approaches based on the Polyakov loop [15-19], the approach based on Fermi liquids theory [20-24] and so on. Compared with the first and second approach, the third one is fundamentally different and powerful. Besides reproducing the EoS accurately, it is also successful in predicting the bulk and transport properties of QGP [20-24].

Gorenstein and Yang pointed out that the initial quasiparticle model was thermodynamically inconsistent and then 
reformulated the statistical mechanics (SM) to solve the inconsistency [25]. But then the expressions of pressure and energy density end up with extra undetermined, temperature dependent terms, which need to be phenomenologically chosen. One should pay attention to this reformulation, which in fact is based on mathematical identities involving derivatives with respect to temperature and chemical potentials, used to redefine the average energy density and the number density, respectively. The qQGP model with reformulated SM by Gorenstein and Yang has been studied by various groups [10-12,26-31]. On the other hand Bannur put forward another method, which skips the thermodynamic inconsistency by avoiding derivatives and instead uses the original definition of all thermodynamic quantities [32]. By doing this, the parameters of qQGP model are reduced. The results of the qQGP model EoS, no matter which SM is adopted, are widely compared with lattice data [27-29,32,33]. The results fit the lattice data well if the parameters are chosen properly.

Besides the EoS and the bulk and transport properties of QGP, quark-number susceptibilities are another important tool to test the reliability of qQGP model $[36,37]$. The second order quark-number susceptibility of finite chemical potential and zero temperature [31] and of finite chemical potential and finite temperature $[38,39]$ are studied. But there are few works on the qQGP model for the higher order susceptibilities associated with the results in RHIC so far. Therefore, in this paper, we will calculate the moments of the baryon distributions of proton and anti-proton in RHIC. By doing this, the study of qQGP model will be improved.

\section{Moments by quasi-particle model}

As mentioned in Ref. [25], since the thermal mass of quasiparticle is temperature and chemical potential related, derivatives of the partition function with respect to temperature and chemical potentials destroy the thermodynamic consistence in the qQGP model. Then we have to redefine the average energy density and number density, respectively, by introducing extra undetermined, temperature dependent terms. Since the common method to obtain the susceptibilities of the baryon number involves derivatives of the partition function with respect to baryon chemical potentials, an extra term must be introduced in the calculation to maintain thermodynamic consistence. To avoid it, we adopt the same method as Bannur has done. In Ref. [32], Bannur gets the expectation of particle number,

$\langle N\rangle=\sum_{k} \frac{z \epsilon_{k} e^{-\beta \epsilon_{k}}}{1 \mp z \epsilon_{k} e^{-\beta \epsilon_{k}}}$,

instead of working with the derivatives of the partition function, $\langle N\rangle=T \frac{\partial \operatorname{Ln} Z}{\partial \mu}$,

where $z$ is the fugacity, $\epsilon_{\mathrm{k}}$ is the single particle energy and $Z$ is the partition function of particles (more details can be found in Ref. [32]). Similarly, in this paper we obtain the quark-number susceptibilities thermodynamic consistently by avoiding to use the derivatives of the partition function. It should be emphasized that, rather than the method of Eq. (1), we calculate the mathematical expectations of $\langle N\rangle$ and $\left\langle N^{n}\right\rangle$ directly by the field theory at finite temperature and the chemical potential according to the Lagrangian of quasiquarks.

For simplicity of the calculation, we adopt the quasiparticle model of QGP here. In this model, the interaction of quarks and gluons is treated as an effective mass term [32]. The effective mass of a quark is made up of the rest mass and the thermal mass,

$m^{2}=m_{q 0}^{2}+\sqrt{2} m_{q 0} m_{\mathrm{th}}+m_{\mathrm{th}}^{2}$,

where $m_{q 0}$ is the rest mass of up or down quark, and in this paper $m_{q 0}=6.5 \mathrm{MeV}$. The temperature and chemical potential dependent quark mass $m_{\text {th }}$ is

$m_{\mathrm{th}}^{2}(\mu, T)=\frac{g^{2} T^{2}}{18} \mathcal{N}_{\mathrm{f}}\left(1+\frac{\mu^{2}}{\pi^{2} T^{2}}\right)$,

and $g^{2}$ is related to the two-loop order running coupling constant,

$$
\begin{aligned}
\alpha_{\mathrm{S}}= & \frac{6 \pi}{\left(33-2 \mathcal{N}_{\mathrm{f}}\right) \ln \frac{T}{\Lambda_{T}} \sqrt{1+a \frac{\mu^{2}}{T^{2}}}} \\
& \times\left(1-\frac{3\left(153-19 \mathcal{N}_{\mathrm{f}}\right)}{\left(33-2 \mathcal{N}_{\mathrm{f}}\right)^{2}} \frac{\ln \left(2 \ln \frac{T}{\Lambda_{T}} \sqrt{1+a \frac{\mu^{2}}{T^{2}}}\right)}{\ln \frac{T}{\Lambda_{T}} \sqrt{1+a \frac{\mu^{2}}{T^{2}}}}\right),
\end{aligned}
$$

where $\alpha_{\mathrm{s}}=g^{2} / 4 \pi$. In this paper, only up and down quarks are considered, so $\mathcal{N}_{\mathrm{f}}=2$. For the parameter $a$ one mainly has two options. One is taking it equal to $(1.91 / 2.91)^{2}$ in the calculation of Schneider [34] and the other is taking $(1 / \pi)^{2}$ in the phenomenological model of Letessier and Rafelski [35].

The expectation of the quark number is

$\left\langle N_{\mathrm{q}}\right\rangle=\left\langle N_{\mathrm{u}}\right\rangle+\left\langle N_{\mathrm{d}}\right\rangle=\mathcal{N}_{\mathrm{c}} \mathcal{N}_{\mathrm{f}}\langle N\rangle$,

where $\langle N\rangle$ is the quark-number expectation of one single color and flavor. And the expectation of the baryon number is $\left\langle N_{\mathrm{B}}\right\rangle=\frac{1}{3}\left\langle N_{\mathrm{q}}\right\rangle$. The variance of the quark number is

$$
\begin{aligned}
\left\langle\left(N_{q}-\left\langle N_{q}\right\rangle\right)^{2}\right\rangle & =\left\langle\left[\left(N_{u}+N_{d}\right)-\left\langle N_{u}+N_{d}\right\rangle\right]^{2}\right\rangle \\
& =\left\langle\left(N_{u}-\left\langle N_{u}\right\rangle\right)^{2}\right\rangle+\left\langle\left(N_{d}-\left\langle N_{d}\right\rangle\right)^{2}\right\rangle
\end{aligned}
$$




$$
\begin{aligned}
& +2\left\langle\left(N_{u}-\left\langle N_{u}\right\rangle\right)\left(N_{d}-\left\langle N_{d}\right\rangle\right)\right\rangle \\
= & \left\langle\left(N_{u}-\left\langle N_{u}\right\rangle\right)^{2}\right\rangle+\left\langle\left(N_{d}-\left\langle N_{d}\right\rangle\right)^{2}\right\rangle \\
= & \mathcal{N}_{c}^{2} \mathcal{N}_{f}\left\langle(N-\langle N\rangle)^{2}\right\rangle,
\end{aligned}
$$

since the up quarks and down quarks are independent, we have $\left\langle\left(N_{\mathrm{u}}-\left\langle N_{\mathrm{u}}\right\rangle\right)\left(N_{\mathrm{d}}-\left\langle N_{\mathrm{d}}\right\rangle\right)\right\rangle=0$. Then the variance of the baryon number is

$$
\begin{aligned}
\sigma^{2} & =\left\langle\left(N_{\mathrm{B}}-\left\langle N_{\mathrm{B}}\right\rangle\right)^{2}\right\rangle \\
& =\frac{1}{\mathcal{N}_{c}^{2}}\left\langle\left(N_{\mathrm{q}}-\left\langle N_{\mathrm{q}}\right\rangle\right)^{2}\right\rangle=\mathcal{N}_{\mathrm{f}}\left\langle(N-\langle N\rangle)^{2}\right\rangle .
\end{aligned}
$$

The skewness of the baryon number is

$$
S=\frac{\left\langle\left(N_{\mathrm{B}}-\left\langle N_{\mathrm{B}}\right\rangle\right)^{3}\right\rangle}{\left[\sigma^{2}\right]^{3 / 2}}=\frac{\mathcal{N}_{\mathrm{f}}\left\langle(N-\langle N\rangle)^{3}\right\rangle}{\left[\mathcal{N}_{\mathrm{f}}\left\langle(N-\langle N\rangle)^{2}\right\rangle\right]^{3 / 2}},
$$

and the kurtosis of the baryon number is

$$
\begin{aligned}
\kappa & =\frac{\left\langle\left(N_{\mathrm{B}}-\left\langle N_{\mathrm{B}}\right\rangle\right)^{4}\right\rangle}{\left[\sigma^{2}\right]^{2}}-3 \\
& =\frac{\mathcal{N}_{\mathrm{f}}\left(\left\langle(N-\langle N\rangle)^{4}\right\rangle+3\left\langle(N-\langle N\rangle)^{2}\right\rangle^{2}\right)}{\left[\mathcal{N}_{\mathrm{f}}\left\langle(N-\langle N\rangle)^{2}\right\rangle\right]^{2}}-3 \\
& =\frac{\mathcal{N}_{\mathrm{f}}\left(\left\langle(N-\langle N\rangle)^{4}\right\rangle-3\left\langle(N-\langle N\rangle)^{2}\right\rangle^{2}\right)}{\left[\mathcal{N}_{\mathrm{f}}\left\langle(N-\langle N\rangle)^{2}\right\rangle\right]^{2}} ;
\end{aligned}
$$

$\beta N=\int_{0}^{\beta} \mathrm{d} \tau \int \mathrm{d}^{3} x \bar{\psi}_{\mathrm{q}} \gamma_{4} \psi_{\mathrm{q}}$

and the quark-number expectation $\langle N\rangle$ is

$$
\begin{aligned}
\langle N\rangle & =\frac{\int \mathcal{D} \bar{\psi}_{\mathrm{q}} \int \mathcal{D} \psi_{\mathrm{q}} \int_{0}^{\beta} \mathrm{d} \tau \int \mathrm{d}^{3} x \bar{\psi}_{\mathrm{q}}(\vec{x}, \tau) \gamma_{4} \psi_{\mathrm{q}}(\vec{x}, \tau) \exp (-\beta(H-\mu N))}{\beta \int \mathcal{D} \bar{\psi}_{\mathrm{q}} \int \mathcal{D} \psi_{\mathrm{q}} \exp (-\beta(H-\mu N))} \\
& =-V T \sum_{k=-\infty}^{+\infty} \int \frac{\mathrm{d}^{3} p}{(2 \pi)^{3}} \operatorname{Tr}\left[G\left(\widetilde{p}_{k}\right) \gamma_{4}\right] \\
& =V T \sum_{k=-\infty}^{+\infty} \int \frac{\mathrm{d}^{3} p}{(2 \pi)^{3}} \frac{4 i \widetilde{\omega}_{k}}{\vec{p}^{2}+m^{2}+\widetilde{\omega}_{k}^{2}},
\end{aligned}
$$

and this expression of the quark number is the same as that widely used in other work [40,41], where $\widetilde{p}_{k}=\left(\vec{p}, \widetilde{\omega}_{k}\right)=$ $\left(\vec{p}, i \mu+\omega_{k}\right), \omega_{k}=(2 k+1) \pi T \cdot \bar{\psi}_{\mathrm{q}} \gamma_{4} \psi_{\mathrm{q}}$ can be analogized as the interaction term. Then the Feynman rules are as follows [42].

1. The vertex is $\gamma_{4}$;

2. the fermion line is $T \sum_{k} \int \frac{\mathrm{d}^{3} p}{(2 \pi)^{3}} G\left(\widetilde{p}_{k}\right)$;

3. we have $-\operatorname{Tr}$ for each closed fermion loop;

4. we have $\beta(2 \pi)^{3} \delta\left(\vec{p}_{\text {in }}-\vec{p}_{\text {out }}\right) \delta_{\omega_{\text {in }}, \omega_{\text {out }}}$ for each vertex, corresponding to energy-momentum conservation; also $\beta(2 \pi)^{3} \delta(0)=\beta V$.

Similarly, the expectation of $N^{2}$ can be expressed as

$$
\left\langle N^{2}\right\rangle=\frac{\int \mathcal{D} \bar{\psi}_{\mathrm{q}} \int \mathcal{D} \psi_{\mathrm{q}} \int_{0}^{\beta} \mathrm{d} \tau_{1} \int \mathrm{d}^{3} x_{1} \bar{\psi}_{q 1} \gamma_{4} \psi_{q 1} \int_{0}^{\beta} \mathrm{d} \tau_{2} \int \mathrm{d}^{3} x_{2} \bar{\psi}_{q 2} \gamma_{4} \psi_{q 2} \exp (-\beta(H-\mu N))}{\beta^{2} * \int \mathcal{D} \bar{\psi}_{\mathrm{q}} \int \mathcal{D} \psi_{\mathrm{q}} \exp (-\beta(H-\mu N))},
$$

then the products of the moments constructed as the experimental observables, $S \sigma$ and $\kappa \sigma^{2}$, are

$$
\begin{aligned}
& S \sigma=\frac{\left\langle(N-\langle N\rangle)^{3}\right\rangle}{\left\langle(N-\langle N\rangle)^{2}\right\rangle}, \\
& \kappa \sigma^{2}=\frac{\left\langle(N-\langle N\rangle)^{4}\right\rangle-3\left\langle(N-\langle N\rangle)^{2}\right\rangle^{2}}{\left\langle(N-\langle N\rangle)^{2}\right\rangle} .
\end{aligned}
$$

Since the quarks are treated as the free quasi-particle with thermal masses, it can be written as

$$
\begin{aligned}
& -\beta(H-\mu N) \\
& \quad=\int_{0}^{\beta} \mathrm{d} \tau \int \mathrm{d}^{3} x \bar{\psi}_{\mathrm{q}}\left(-\gamma_{4} \frac{\partial}{\partial \tau}+i \vec{\gamma} \cdot \vec{\nabla}-m+\mu \gamma_{4}\right) \psi_{\mathrm{q}},
\end{aligned}
$$

where $\psi_{\mathrm{q}}$ is the quark field and $\mu$ is the chemical potential of quarks $\left(\mu=\mu_{\mathrm{u}}=\mu_{d}=\frac{1}{3} \mu_{\mathrm{B}}\right)$, then the quark number $N$ is and the Feynman diagram for

$\frac{\int \mathcal{D} \bar{\psi}_{\mathrm{q}} \int \mathcal{D} \psi_{\mathrm{q}} \int_{0}^{\beta} \mathrm{d} \tau_{1} \int \mathrm{d}^{3} x_{1} \bar{\psi}_{11} \gamma_{4} \psi_{q 1} \int_{0}^{\beta} \mathrm{d} \tau_{2} \int \mathrm{d}^{3} x_{2} \bar{\psi}_{q 2} \gamma_{4} \psi_{q 2} \exp (-\beta(H-\mu N))}{\left(\int \mathcal{D} \bar{\psi}_{\mathrm{q}} \int \mathcal{D} \psi_{\mathrm{q}} \exp (-\beta(H-\mu N))\right.}$ is shown in Fig. 1. Then the variance of $N$ is

$$
\begin{aligned}
\left\langle(N-\langle N\rangle)^{2}\right\rangle & =\left\langle N^{2}\right\rangle-\langle N\rangle^{2} \\
& =-V T^{2} \sum_{k=-\infty}^{+\infty} \int \frac{\mathrm{d}^{3} p}{(2 \pi)^{3}} \operatorname{Tr}\left[G\left(\widetilde{p}_{k}\right) \gamma_{4} G\left(\widetilde{p}_{k}\right) \gamma_{4}\right] \\
& =-V T^{2} \sum_{k=-\infty}^{+\infty} \int \frac{\mathrm{d}^{3} p}{(2 \pi)^{3}} \frac{4\left(\vec{p}^{2}+m^{2}-\widetilde{\omega}_{k}^{2}\right)}{\left(\vec{p}^{2}+m^{2}+\widetilde{\omega}_{k}^{2}\right)^{2}} .
\end{aligned}
$$

The Feynman diagram for $\left\langle N^{3}\right\rangle$ is shown in Fig. 2 and the third moment of $N$ is

$$
\begin{aligned}
& \left\langle(N-\langle N\rangle)^{3}\right\rangle=\left\langle N^{3}\right\rangle-3\langle N\rangle\left\langle N^{2}\right\rangle+2\langle N\rangle^{3} \\
& =-2 V T^{3} \sum_{k=-\infty}^{+\infty} \int \frac{\mathrm{d}^{3} p}{(2 \pi)^{3}} \operatorname{Tr}\left[G\left(\widetilde{p}_{k}\right) \gamma_{4} G\left(\widetilde{p}_{k}\right) \gamma_{4} G\left(\widetilde{p}_{k}\right) \gamma_{4}\right] \\
& =2 V T^{3} \sum_{k=-\infty}^{+\infty} \int \frac{\mathrm{d}^{3} p}{(2 \pi)^{3}} \frac{4 i \widetilde{\omega}_{k}\left(3 \vec{p}^{2}+3 m^{2}-\widetilde{\omega}_{k}^{2}\right)}{\left(\vec{p}^{2}+m^{2}+\widetilde{\omega}_{k}^{2}\right)^{3}} .
\end{aligned}
$$




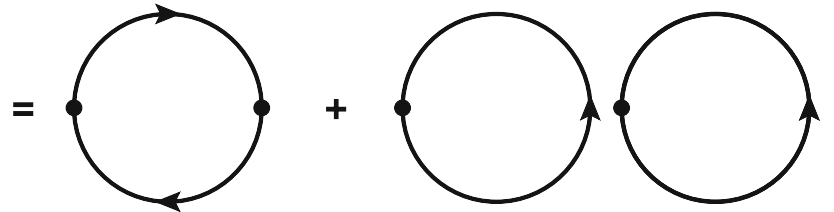

Fig. 1 Feynman diagram for $\left\langle N^{2}\right\rangle$. The point represents the vertex and the line represents the quark propagator

The Feynman diagram for $\left\langle N^{4}\right\rangle$ is shown in Fig. 3 and the numerator of $\kappa \sigma^{2}$ is

$$
\begin{aligned}
\langle(N- & \left.\langle N\rangle)^{4}\right\rangle-3\left\langle(N-\langle N\rangle)^{2}\right\rangle^{2} \\
= & -6 V T^{4} \\
& \times \sum_{k=-\infty}^{+\infty} \int \frac{\mathrm{d}^{3} p}{(2 \pi)^{3}} \operatorname{Tr}\left[G\left(\widetilde{p}_{k}\right) \gamma_{4} G\left(\widetilde{p}_{k}\right) \gamma_{4} G\left(\widetilde{p}_{k}\right) \gamma_{4} G\left(\widetilde{p}_{k}\right) \gamma_{4}\right] \\
= & -6 V T^{4} \\
& \times \sum_{k=-\infty}^{+\infty} \int \frac{\mathrm{d}^{3} p}{(2 \pi)^{3}} \frac{4\left(\left(\vec{p}^{2}+m^{2}\right)^{2}-6 \widetilde{\omega}_{k}^{2}\left(\vec{p}^{2}+m^{2}\right)+\widetilde{\omega}_{k}^{4}\right)}{\left(\vec{p}^{2}+m^{2}+\widetilde{\omega}_{k}^{2}\right)^{4}} .
\end{aligned}
$$

Putting the results of Eqs. (17) and (18) into (11), we get the value of $S \sigma$,

$$
S \sigma=\frac{2 T \sum_{k=-\infty}^{+\infty} \int \frac{\mathrm{d}^{3} p}{(2 \pi)^{3}} \operatorname{Tr}\left[G\left(\widetilde{p}_{k}\right) \gamma_{4} G\left(\widetilde{p}_{k}\right) \gamma_{4} G\left(\widetilde{p}_{k}\right) \gamma_{4}\right]}{\sum_{k=-\infty}^{+\infty} \int \frac{\mathrm{d}^{3} p}{(2 \pi)^{3}} \operatorname{Tr}\left[G\left(\widetilde{p}_{k}\right) \gamma_{4} G\left(\widetilde{p}_{k}\right) \gamma_{4}\right]},
$$

and putting the results of Eqs. (18) and (19) into (12), we get $\kappa \sigma^{2}$,

$$
\kappa \sigma^{2}=\frac{3 T^{2} \sum_{k=-\infty}^{+\infty} \int \frac{\mathrm{d}^{3} p}{(2 \pi)^{3}} \operatorname{Tr}\left[G\left(\widetilde{p}_{k}\right) \gamma_{4} G\left(\widetilde{p}_{k}\right) \gamma_{4} G\left(\widetilde{p}_{k}\right) \gamma_{4} G\left(\widetilde{p}_{k}\right) \gamma_{4}\right]}{\sum_{k=-\infty}^{+\infty} \int \frac{d^{3} p}{(2 \pi)^{3}} \operatorname{Tr}\left[G\left(\widetilde{p}_{k}\right) \gamma_{4} G\left(\widetilde{p}_{k}\right) \gamma_{4}\right]} .
$$

\section{Results}

The experimental results for the $S \sigma$ and $\kappa \sigma^{2}$ of net-proton multiplicity distributions are shown in Figs. 4 and 5, respectively. In Fig. 4, the top three lines are $S \sigma$ results from Eq. (11) as a function of $\sqrt{S_{N N}}$ for $\Lambda_{T} / T_{\mathrm{c}}=0.85,0.7,0.6$ at $a=(1.91 / 2.91)^{2}$ and the bottom three lines are for $\Lambda_{T} / T_{\mathrm{c}}=0.7,0.63,0.58$ at $a=(1 / \pi)^{2}$, where $T_{\mathrm{c}}=175$ $\mathrm{MeV}$ is from Ref. [4]. The temperature and baryon chemical potential parameters for each energy are determined from the chemical freeze-out parameterization in heavy-ion collisions [43]. Data points are the experimental results of $S \sigma$ from Ref. $[7,8]$. In Fig. 5, the top three lines are $\kappa \sigma^{2}$ results from Eq. (12) as a function of $\sqrt{S_{N N}}$ for $\Lambda_{T} / T_{\mathrm{c}}=0.85,0.7,0.6$ at $a=(1.91 / 2.91)^{2}$ and the bottom three lines are for $\Lambda_{T} / T_{\mathrm{c}}=0.7,0.63,0.57$ at $a=(1 / \pi)^{2}$. Data points from Ref. $[7,8]$ are $\kappa \sigma^{2}$ results of $\mathrm{Au}+\mathrm{Au}$ collisions at different centrality bins.

There are two parameters $a$ and $\Lambda_{T}$ in our calculation. The parameter $a$ is introduced to take account of the finite quark chemical potential $\mu[34,35]$. As mentioned above, there are mainly two options: $a=(1.91 / 2.91)^{2}$ [34] and $a=(1 / \pi)^{2}[35] . \Lambda_{T}$ is related to the QCD scale parameter. Since the second order quark-number susceptibility is studied at $\Lambda_{T} / T_{\mathrm{c}}=0.7$ [38], $S \sigma$ and $\kappa \sigma^{2}$ are calculated with $\Lambda_{T} / T_{\mathrm{c}}$ around 0.7. When the parameter $a$ is fixed, the values of $S \sigma$

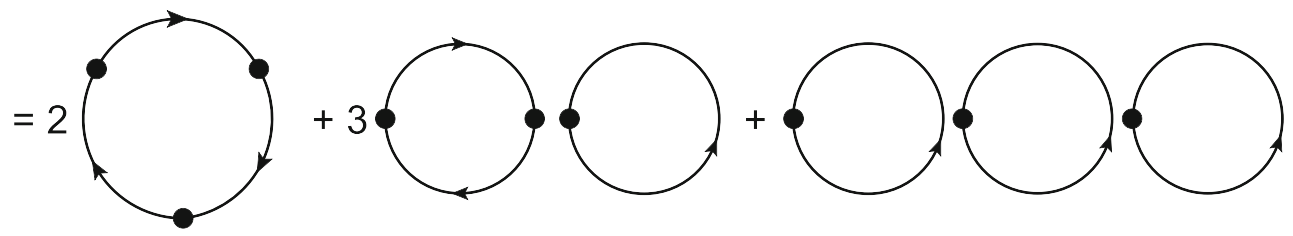

Fig. 2 Feynman diagram for $\left\langle N^{3}\right\rangle$. The point represents the vertex and the line represents the quark propagator
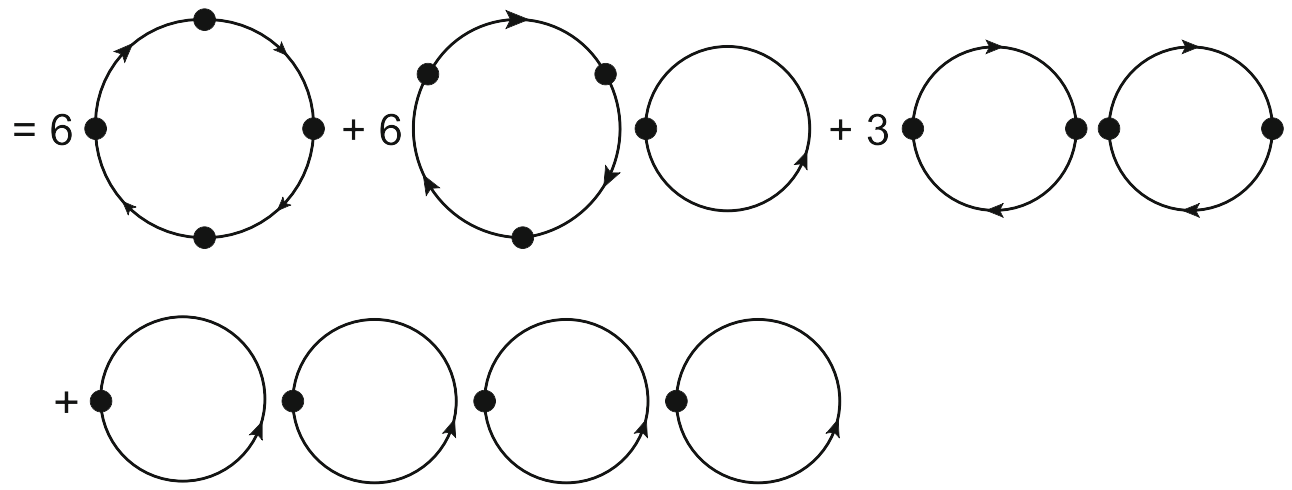

Fig. 3 Feynman diagram for $\left\langle N^{4}\right\rangle$. The point represents the vertex and the line represents the quark propagator 


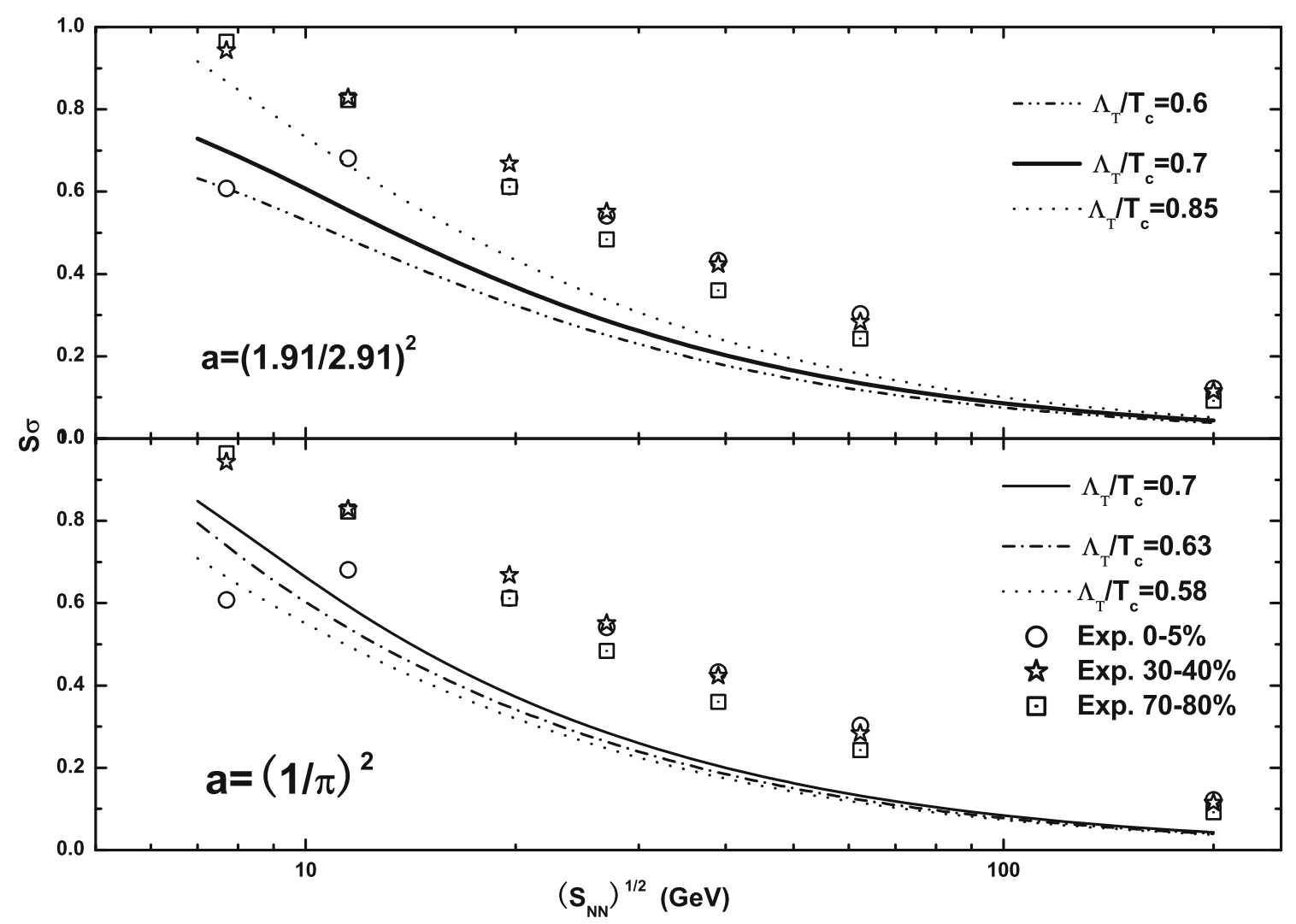

Fig. 4 The top three lines are $S \sigma$ results from Eq. (11) as a function of $\sqrt{S_{N N}}$ for $\Lambda_{T} / T_{\mathrm{c}}=0.85,0.7,0.6$ at $a=(1.91 / 2.91)^{2}$. The bottom three lines are $S \sigma$ for $\Lambda_{T} / T_{\mathrm{c}}=0.7,0.63,0.58$ at $a=(1 / \pi)^{2}$. Data points from Ref. [7,8] are $S \sigma$ results for $0-5 \%, 30-40 \%$ and $70-80 \%$ Au+Au collisions, respectively

and $\kappa \sigma^{2}$ are reduced with the reduction of $\Lambda_{T}$. In Fig. 4 the difference between the results of $S \sigma$ calculated from different $\Lambda_{T}$ are smaller at high energies than low energies. For $\kappa \sigma^{2}$ in Fig. 5 the results at different $\Lambda_{T}$ are almost parallel with each other at large $\sqrt{S_{N N}}$ and they have with bigger discrepancies at small $\sqrt{S_{N N}}$.

Particularly, the results for $\Lambda_{T} / T_{\mathrm{c}}=0.7$ are shown as solid lines in Figs. 4 and 5. Comparing the two solid lines in Fig. 4, we find that the values with $a=(1.91 / 2.91)^{2}$ are lower than the one with $a=(1 / \pi)^{2}$ at small $\sqrt{S_{N N}}$ and the difference gets smaller and smaller with increasing $\sqrt{S_{N N}}$. As for $\kappa \sigma^{2}$ in Fig. 5, at small $\sqrt{S_{N N}}$ the two lines have different trends. The one with $a=(1.91 / 2.91)^{2}$ increases with increasing $\sqrt{S_{N N}}$ and the other one shows an opposite trend. The results of the qQGP model are more sensitive to the parameters at small colliding energies.

The experimental results in Figs. 4 and 5 demonstrate that both $S \sigma$ and $\kappa \sigma^{2}$ clearly show a non-monotonic variation for $0-5 \%$ centrality when $\sqrt{S_{N N}}$ is below $30 \mathrm{GeV}$. Above $30 \mathrm{GeV}$ the results of different centralities are close to each other. The experimental results indicate that the corresponding chemical freeze-out $\mathrm{T}$ and $\mu$ around $20 \mathrm{GeV}$ may be close to the critical point $[7,8]$. In Figs. 4 and 5, it is shown that our results with different parameters show similar trends to the experimental data for the colliding energies above 30 $\mathrm{GeV}$. In this region, for $S \sigma$ our results is approximately 0.1 less than the experimental data at the maximum deviation, and for $\kappa \sigma^{2}$ our results describe the experimental data well. But below $30 \mathrm{GeV}$, our results have significant discrepancies from the experimental data of $0-5 \%$ centrality.

\section{Summary}

Baryon number fluctuations are sensitive to the QCD phase transition and QCD critical point. We calculated various order moments of the baryon number distributions in the quasi-particle model of QGP. To avoid an extra undetermined term in calculating susceptibilities in the quasi-particle model we try to directly calculate the various order of moments of the quark-number distributions. Since the term of the quark number in the Lagrangian is analogized as the interaction term, we can obtain the moments of the quark number based on the Feynman rules of finite-temperature field theory. Finally, we compare our calculations with the latest experimental data. It is found that the results of qQGP model 


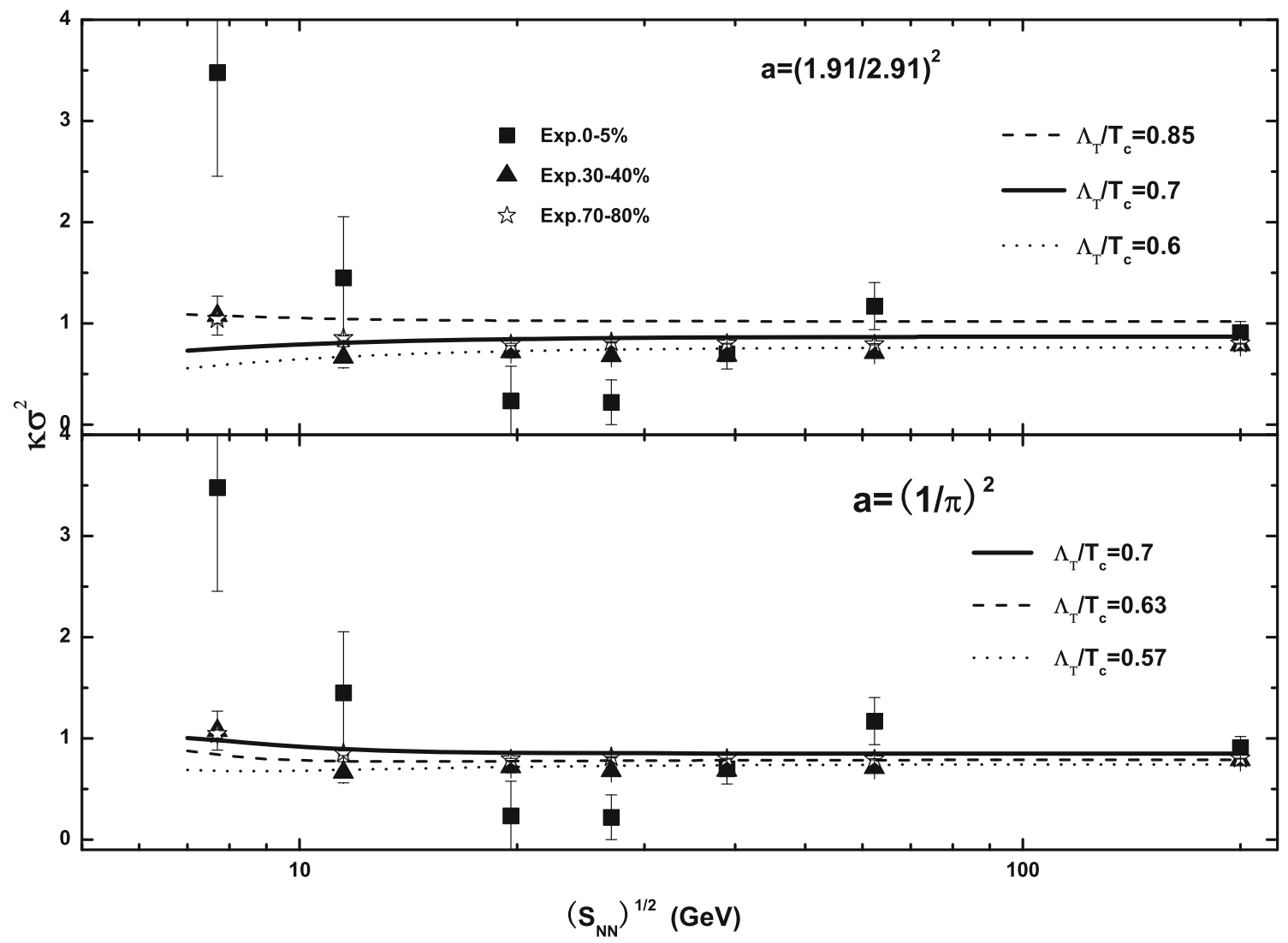

Fig. 5 The top three lines are $\kappa \sigma^{2}$ results from Eq. (12) as a function of $\sqrt{S_{N N}}$ for $\Lambda_{T} / T_{\mathrm{c}}=0.85,0.7,0.6$ at $a=(1.91 / 2.91)^{2}$. The bottom three lines are $\kappa \sigma^{2}$ for $\Lambda_{T} / T_{\mathrm{c}}=0.7,0.63,0.57$ at $a=(1 / \pi)^{2}$. Data points from Ref. [7,8] are $\kappa \sigma^{2}$ results of Au+Au collisions at different centrality bins

are more sensitive to the parameters at small colliding energies. For energies above $30 \mathrm{GeV}$, our results with different parameters show similar trends to the experimental data. We found that the $S \sigma$ are smaller than the experimental data, while $\kappa \sigma^{2}$ fits the experimental data well. However, at energies below $30 \mathrm{GeV}$, our results have large discrepancies from the experimental data of $0-5 \%$ centrality. These comparisons suggest that, at low energies, the experimental data may contain other physics effects, for e.g. the critical point, which is not included in the qQGP model. It also indicates that the future low energy heavy-ion collisions experiment is much more important for the QCD critical point search.

Acknowledgements This work was supported by the MoST of China 973-Project No. 2015CB856901 and the National Natural Science Foundation of China (under Grants No. 11447121, 11575069, 11475085, 11690030, and No. 11535005).

Open Access This article is distributed under the terms of the Creative Commons Attribution 4.0 International License (http://creativecomm ons.org/licenses/by/4.0/), which permits unrestricted use, distribution, and reproduction in any medium, provided you give appropriate credit to the original author(s) and the source, provide a link to the Creative Commons license, and indicate if changes were made. Funded by SCOAP ${ }^{3}$.

\section{References}

1. M.M. Aggarwal et al., Phys. Rev. Lett. 105, 022302 (2010)

2. M.A. Stephanov, Phys. Rev. Lett. 102, 032301 (2009)

3. C. Athanasiou et al., Phys. Rev. D 82, 074008 (2010)

4. S. Gupta et al., Science 332, 1525 (2011)

5. L. Adamczyk et al., Phys. Rev. Lett. 112, 032302 (2014)

6. M.A. Stephanov, J. Phys. G 38(1), 24147 (2011)

7. X. Luo, PoS(CPOD2014)019. arXiv: 1503.02558

8. X. Luo, Nucl. Phys. A, 1-9 (2016). arXiv:1512.09215

9. A. Peshier, B. Kampfer, O.P. Pavlenko, G. Soff, Phys. Lett. B 337, 235 (1994)

10. A. Peshier, B. Kampfer, G. Soff, Phys. Rev. C 61, 045203 (2000)

11. A. Peshier, B. Kampfer, G. Soff, Phys. Rev. D 66, 094003 (2002)

12. A. Peshier et al., Phys. Rev. D 54, 2399 (1996)

13. C.R. Allton et al., Phys. Rev. D 68, 014507 (2003)

14. C.R. Allton et al., Phys. Rev. D 71, 054508 (2005)

15. A. Dumitru, R.D. Pisarski, Phys. Lett. B 525, 95 (2002)

16. K. Fukushima, Phys. Lett. B 591, 277 (2004)

17. S.K. Ghosh et al., Phys. Rev. D 73, 114007 (2006)

18. H. Abuki, K. Fukushima, Phys. Lett. B 676, 57 (2006)

19. H.M. Tsai, B. Müller, J. Phys. G 36, 075101 (2009)

20. V. Chandra, V. Ravishankar, Nucl. Phys. A 848, 330 (2010)

21. V. Chandra, V. Ravishankar. Euro. Phys. J C 59, 705 (2009)

22. V. Chandra, R. Kumar, V. Ravishankar. Phys. Rev. C 76, 054909 (2007)

23. V. Chandra, A. Ranjan, V. Ravishankar, Euro. Phys. J A 40, 109 (2009) 
24. V. Chandra, V. Ravishankar. Phys. Rev. D 84, 074013 (2011)

25. M.I. Gorenstein, S.N. Yang, Phys. Rev. D 52, 5206 (1995)

26. P. Levai, U. Heinz, Phys. Rev. C 57, 1879 (1998)

27. R.A. Schneider, W. Weise, Phys. Rev. C 64, 055201 (2001)

28. K.K. Szabo, A.I. Toth, JHEP 0306, 008 (2003)

29. Y.B. Ivanov, V.V. Skokov, V.D. Toneev, Phys. Rev. D 71, 014005 (2005)

30. J. Cao, Y. Jiang, W.M. Sun, H.S. Zong, Phys. Lett. B 771, 65 (2012)

31. L.J. Luo, J. Cao, Y. Yan, W.M. Sun, H.S. Zong, Eur. Phys. J. C 73, $2626(2013)$

32. V.M. Bannur, JHEP 0709, 046 (2007)

33. B. Kampfer, A. Peshier, G. Soff, arXiv:hep-ph/0212179

34. R.A. Schneider, arXiv:hep-ph/0303104

35. J. Letessier, J. Rafelski, Phys. Rev. C 67, 031902 (2003)
36. M. Bluhm, B. Kämpfer, G. Soff, Phys. Lett. B 620, 131 (2005)

37. S. Plumari, W.M. Alberico, V. Greco, C. Ratti, Phys. Rev. D 84, 094004 (2011)

38. J. Cao et al., Chin. Phys. Lett. 27(3), 031201 (2010)

39. H. Berrehrah, W. Cassing, E. Bratkovskaya, T. Steinert, Phys. Rev. C 93, 044914 (2016)

40. H.S. Zong, W.M. Sun, Phys. Rev. D 78, 054001 (2008)

41. M. He, J.F. Li, W.M Sun, H.S. Zong. Phys. Rev. D 79, 036001 (2009)

42. J.I. Kapusta, C. Gale, Finite-Temperature Field Theory Principles and Applications (Cambridge University Press, Cambridge, 2006)

43. J. Cleymans, H. Oeschler, K. Redlich, S. Wheaton, Phys. Rev. C 73, $034905(2006)$ 